\title{
High-level expression of a novel liver-targeting fusion interferon with preferred Escherichia coli codon preference and its anti-hepatitis $B$ virus activity in vivo
}

Xuemei $\mathrm{Lu}^{1,2^{*}}$, Jie Wang ${ }^{1,2}$, Xiaobao Jin ${ }^{1,2}$ and Jiayong Zhu $\mathrm{u}^{1,2^{*}}$

\begin{abstract}
Background: In our previous study, a novel liver-targeting fusion interferon (IFN-CSP) combining IFN a2b with plasmodium region I peptide was successfully constructed. IFN-CSP has significant inhibition effects on HBV-DNA replication in HepG2.2.15 cells. The aim of the present investigation was focused on how to produce high levels of recombinant IFN-CSP and its in vivo anti-hepatitis B virus (HBV) activity.

Methods: A modified DNA fragment encoding IFN-CSP was synthesized according to Escherichia coli (E. coli) preferred codon usage and transformed into E. coli BL21 (DE3) for protein expression. The induction conditions were systematically examined by combining one-factor experiments with an orthogonal test $(L(9)(3)(4))$. The antigenicity of the purified protein was characterized by western blot analysis. The in vivo tissue distribution were assayed and compared with native IFN a2b. HBV-transgenic mice were used as in vivo model to evaluate the anti-HBV effect of the recombinant IFN-CSP.

Results: The results showed that the E. coli expression system was very efficient to produce target protein. Conclusion: Our current research demonstrates for the first time that IFN-CSP gene can be expressed at high levels in E. coli through codon and expression conditions optimization. The purified recombinant IFN-CSP showed liver-targeting potentiality and anti-HBV activity in vivo. The present study further supported the application of IFN-CSP in liver-targeting anti-HBV medicines.
\end{abstract}

Keywords: Preferred codon usage, Induction conditions optimize, Recombinant liver-targeting fusion interferon, HBV transgenic mice

\section{Background}

Over the past decade, a number of functional proteins have been produced successfully by recombinant DNA methods [1,2]. The most commonly used host cell is Escherichia coli (E. coli) because of high yield and low cost [3]. However, high-level expression of recombinant proteins using E. coli as the host cell has been affected

\footnotetext{
*Correspondence: luxuemei605@163.com; zhujiayong1020@163.com

'School of Basic Courses, Guangdong Pharmaceutical University, 280 Wai Huan Dong Road, Guangzhou Higher Education Mega Center, Guangzhou, People's Republic of China

${ }^{2}$ Guangdong Provincial Key Laboratory of Pharmaceutical Bioactive Substances, 280 Wai Huan Dong Road, Guangzhou Higher Education Mega Center, Guangzhou, People's Republic of China
}

by some factors, such as the different preferential codons in different expression systems and various induction conditions of target protein $[4,5]$.

The interferons (IFNs) are a family of protein with the ability to induce antiproliferative, immunomodulatory, and antiviral activities [6-8]. For more than a decade, interferon therapy is the gold standard in treatment for certain forms of viral hepatitis and carcinogenesis [9]. However, therapeutic efficacy has been limited because interferon does not have organ-specific affinity and its in vivo half-life is short [10]. Incorporation of plasmodium region I peptide was demonstrated to be a promising strategy for the development of liver-targeting drug $[11,12]$. In our previous study, a novel liver-targeting



BïoMed Central
(C) 2015 Lu et al. This is an Open Access article distributed under the terms of the Creative Commons Attribution License (http://creativecommons.org/licenses/by/4.0), which permits unrestricted use, distribution, and reproduction in any medium, provided the original work is properly credited. The Creative Commons Public Domain Dedication waiver (http:// creativecommons.org/publicdomain/zero/1.0/) applies to the data made available in this article, unless otherwise stated. 
fusion interferon (IFN-CSP) combining IFN $\alpha 2 b$ with plasmodium region I peptide was successfully designed [13]. The results of in vitro anti-hepatitis B virus (HBV) activity of IFN-CSP showed that IFN-CSP has significant inhibition effects on HBV-DNA replication in HepG2.2.15 cells [14]. In vitro liver tissue binding analysis revealed that IFN-CSP specific targeting to liver tissue [13]. IFN-CSP may be an excellent candidate as a liver-targeting antiHBV agent. However, the in vivo tissue distribution and anti-HBV activity of IFN-CSP requires further investigate. Moreover, it is desirable to produce IFN-CSP in a large scale for therapeutic application.

The main purpose of the present investigation was focused on how to produce high levels of recombinant IFN-CSP in a cost-effective way. We also investigated the in vivo tissue distribution and anti-HBV activity of IFN-CSP. Therefore, a modified DNA fragment encoding the IFN-CSP was synthesized by overlapping extension-PCR method according to E. coli preferred codon usage. The IFN-CSP gene was cloned into the bacterial expression vector pET-21b and transferred into the expression strain E. coli BL21. The suitable induction conditions were systematically optimized by combining one-factor experiments with an orthogonal experiment $(\mathrm{L}(9)(3)(4))$. The antigenicity of the purified protein was characterized by western blot analysis. The in vivo tissue distribution were assayed and compared with native IFN $\alpha 2 b$. HBV-transgenic mice were used as in vivo model to evaluate the anti-HBV effect of the recombinant IFN-CSP.

\section{Methods}

Pasmids, strains and culture media

pMD20-T (Takara, Japan) was used for gene cloning. pET-21b (Novagen, USA) was employed to construct expression vector. $E$. coli strain DH5 $\alpha$ (Novagen, USA) was applied as the host for gene manipulation. E. coli strain BL21 (DE3; Novagen, USA) served as expression host for fusion protein. Luria-Bertani (LB) medium was used for bacterial growth and protein expression.

\section{Construction of the liver-targeting fusion interferon gene using $E$. coli preferred codon}

To improve the expression level of IFN-CSP in E. coli, a new IFN-CSP coding sequence was designed. The optimized codons usage pattern of $E$. coli genes was employed according to the codon usage pattern of E. coli (http:// www.kazusa.or.jp/codon/). Based on the method of polymerase chain reaction (PCR)-based gene synthesis and gene splicing by overlap extension, a modified three-step method [15], called "splicing by overlapping extensionPCR (SOE-PCR)", was adopted to construct the synthetic IFN-CSP gene. We designed 16 oligonucleotides encoding for the IFN-CSP protein. The sense and antisense oligonucleotides with 19 bp complementary overlapping sequence are presented in Table 1.

\section{Construction of expression plasmids IFN-CSP/pET-21b}

The synthetic IFN-CSP gene fragments were cloned into the pMD20-T vector (Takara) and transformed into E. coli DH5a according to the procedures described by the manufacturer.

Table 1 Nucleotide sequences of oligonucleotides designed for assembly of IFN-CSP

\begin{tabular}{|c|c|c|}
\hline Primers & Location & Nucleotide sequence (from $5^{\prime}$ end to $3^{\prime}$ end) \\
\hline$\overline{\mathrm{SR}-1}$ & $1-29$ & GGAATTCCATATGTGTGATCTGCCTCAAA \\
\hline AR-1 & $14-71$ & GTGCCAGGAGCATCAAGGTACGACGGCTACCCAGGCTGTGGGTTTGAGGCAGATCACA \\
\hline SR-2 & $53-110$ & ACCTTGATGCTCCTGGCACAGATGCGTCGTATCTCTCTTTTCTCCTGCTTGAAGGACC \\
\hline AR-2 & $92-149$ & GGTTGCCAAACTCCTCCTGTGGAAATCCAAAGTCATGACGGTCCTTCAAGCAGGAGAA \\
\hline SR-3 & $131-188$ & CAGGAGGAGTTTGGCAACCAGTTCCAAAAGGCTGAAACCATCCCTGTCCTCCATGAGA \\
\hline AR-3 & $170-227$ & AGTCCTTTGTGCTGAAGAGATTGAAGATCTGCTGGATCATCTCATGGAGGACAGGGAT \\
\hline SR-4 & $209-266$ & CTCTTCAGCACAAAGGACTCATCTGCTGCTTGGGATGAGACCCTCCTTGACAAATTCT \\
\hline AR-4 & 248-305 & AGGCTTCCAGGTCATTCAGCTGCTGGTAGAGTTCAGTGTAGAATTTGTCAAGGAGGGT \\
\hline SR-5 & $287-344$ & CTGAATGACCTGGAAGCCTGTGTGATTCAGGGGGTGGGGGTGACAGAGACTCCACTGA \\
\hline AR-5 & $326-383$ & GGAAGTATTTACGCACAGCCAGAATGGAGTCCTCCTTCATCAGTGGAGTCTCTGTCAC \\
\hline SR-6 & $365-422$ & GCTGTGCGTAAATACTTCCAACGTATCACTCTCTATCTGAAAGAGAAGAAATACAGCC \\
\hline AR-6 & $404-461$ & AACGCATGATTTCTGCACGGACAACCTCCCAGGCACAAGGGCTGTATTTCTTCTCTTT \\
\hline SR-7 & $443-500$ & CGTGCAGAAATCATGCGTTCTITTCTITGTCAACAAACTTGCAAGAAAGTTTACGTA \\
\hline AR-7 & $482-525$ & CGTAATTTCTCGTTGTCTTCCTTACTACGTAAACTTTCTTGCAA \\
\hline SR-8 & $507-561$ & AAGACAACGAGAAATTACGTAAACCAAAACATAAAAAATTAAAGCAACCAGCGGA \\
\hline AR-8 & $545-578$ & CCGCTCGAGATTAACCATCCGCTGGTTGCTTTAA \\
\hline
\end{tabular}


The generated recombinant plasmids IFN-CSP/pMD20-T were digested and the inserts were cloned into Nde I/Xho I restriction sites of the expression vector pET-21b (Fig. 1a). The resulting expression plasmid IFN-CSP/pET-21b was finally transformed into E. coli BL21 (DE3) for IFN-CSP expression.

\section{Optimization of IFN-CSP expression}

To improve the expression level of IFN-CSP, the induction conditions [16] like cultivation temperature, induction timing, inducer concentrations, induction time were systematically examined by combining one-factor experiments with an orthogonal test $(\mathrm{L}(9)(3)(4))$. A fresh clone of recombinant E. coli BL21 with plasmid IFN-CSP/ pET-21b grew in Luria-Bertani (LB) medium containing $100 \mu \mathrm{g} / \mathrm{ml}$ ampicillin. Different conditions of induction (temperatures: $17{ }^{\circ} \mathrm{C}, 22{ }^{\circ} \mathrm{C}, 27^{\circ} \mathrm{C}, 32{ }^{\circ} \mathrm{C}, 37{ }^{\circ} \mathrm{C}, 42{ }^{\circ} \mathrm{C}$; $\mathrm{OD}_{600}: 0.1,0.2,0.4,0.6,0.8,1.0,2.0$; IPTG concentrations: $0.1,0.2,0.4,0.6,0.8,1.0,1.2,1.5 \mathrm{mM}$; induction times: $1,2,4,6,8,10,12,24 \mathrm{~h}$ ) were conducted to optimize the expression of heterologous protein in $E$. coli. To evaluate the expression level of IFN-CSP, the cells were harvested by centrifugation at 10,000 rpm for 10 min. Samples were analyzed by $15 \%$ sodium dodecyl sulfate polyacrylamide gel electrophoresis (SDS-PAGE) and the percentage fraction of proteins was assessed by densitometric using Gel-Pro analyzer Version 4.5 software. Total protein concentration was determined by Bradford method and the concentration of IFN-CSP was calculated according the percentage fraction and total protein concentration.

\section{Purification, antigenicity and biological activity analysis} Cells were collected by centrifugation. After lysis by ultrasonication in an ice bath, an improved seven-step process was conducted to obtain purified IFN-CSP based on previously described methods [13, 15, 17]. Briefly, cellular pellet was separated from the cell lysate by centrifugation at $6000 \mathrm{~g}$ and $4{ }^{\circ} \mathrm{C}$ for $10 \mathrm{~min}$. IB material was washed with $1 \%$ Triton $\mathrm{X}-100$ and $2 \mathrm{M}$ urea (containing $2 \%$ deoxycholate), and then dissolved in $6 \mathrm{M}$ guanidine hydrochloride $(\mathrm{GuHCl})$ (containing $2.5 \mathrm{mM}$ DTT and $50 \mathrm{mM}$ Tris-HCL buffer, $\mathrm{pH}$ 8.0). The solubilized IFN-CSP was refolded by dialysis with the $50 \mathrm{mM}$ Tris- $\mathrm{HCl}$ buffer systems (containing $0.2 \mathrm{mM}$ glutathione oxidized and $2 \mathrm{mM}$ glutathione reduced), followed by changes of the buffer containing decreasing $\mathrm{GuHCl}$ concentrations (4, 2 and $1 \mathrm{M}$ ) and finally with Tris- $\mathrm{HCl}$ buffer $(\mathrm{pH}$ 7.4). The refolded IFN-CSP was purified by heparin affinity chromatography according to the instruction of HiTrap affinity column (GE healthcare, USA). The IFN-CSP was eluted with a linear gradient of 0.1-2 $\mathrm{M} \mathrm{NaCl}$. After dialysis against the Tris- $\mathrm{HCl}$ buffer, the sample was further purified to remove endotoxin by polymyxin B column (Bio-Rad, USA). After detecting the lipopolysaccharides (LPS) content with the chromogenic limulus amoebocyte lysate assay (Associates of Cape Cod, USA), the purified IFN-CSP was lyophilized and stored at $-80{ }^{\circ} \mathrm{C}$.

The antigenicity of the purified protein was characterized by western blot analysis. We performed western blot with primary goat polyclonal anti-IFN $\alpha$ antibody (1:200; Santa Cruz Biotechnology, USA) and peroxidaseconjugated rabbit anti-goat IgG (1:2500; Santa Cruz Biotechnology, USA) as previously described [13]. The purity of the purified protein was assessed by reverse phase high-performance liquid chromatography (RP-HPLC) on a C18 column $(250 \times 4.6 \mathrm{~mm}, 5 \mu \mathrm{m}$, and $300 \AA$, Agilent, USA) in an analytical Alliance HPLC System (Waters,
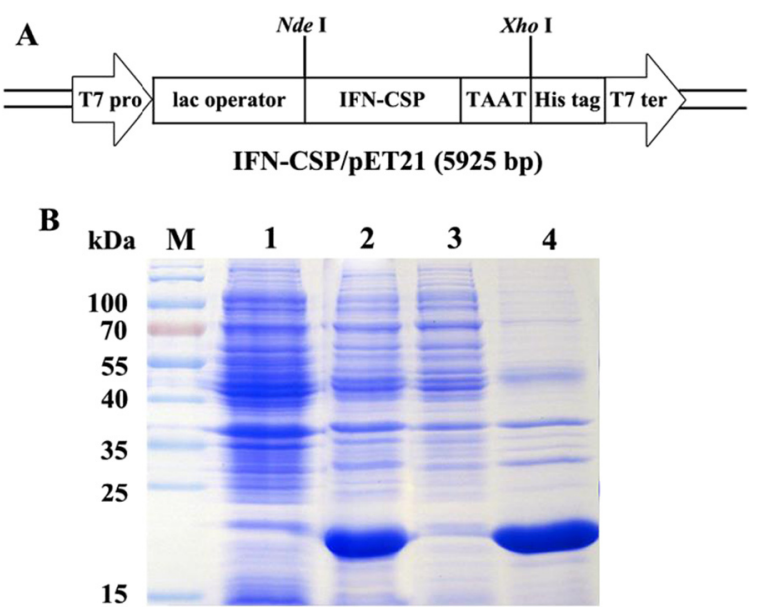

Fig. 1 Schematic diagram of IFN-CSP gene in the expression vector IFN-CSP/pET-21b and expression of IFN-CSP protein in E. coli BL21/pET-21b-IFN-CSP. a: A Schematic diagram of IFN-CSP/pET-21b (T7 pro, T7 promoter; T7 ter, T7 terminator). b: SDS-PAGE analysis of protein expression. Lane M: Protein molecular weight marker, Lane 1-2: Total proteins of E. coli BL21/pET-21 b-IFN-CSP before and after induction, Lane 3-4: Supernatant and precipitation after ultrasonication and centrifugation 
1 TGT GAT CTG CCT CAA ACC CAC AGC CTG GGT AGC CGT CGT ACC TTG

$$
\begin{array}{lllllllllllllll}
\mathbf{C} & \mathbf{D} & \mathbf{L} & \mathbf{P} & \mathbf{Q} & \mathbf{T} & \mathbf{H} & \mathbf{S} & \mathbf{L} & \mathbf{G} & \mathbf{S} & \mathbf{R} & \mathbf{R} & \mathbf{T} & \mathbf{L}
\end{array}
$$

46 ATG CTC CTG GCA CAG ATG CGT CGT ATC TCT CTT TTC TCC TGC TTG

$\begin{array}{lllllllllllllll}\mathbf{M} & \mathbf{L} & \mathbf{L} & \mathbf{A} & \mathbf{Q} & \mathbf{M} & \mathbf{R} & \mathbf{R} & \mathbf{I} & \mathbf{S} & \mathbf{L} & \mathbf{F} & \mathbf{S} & \mathbf{C} & \mathbf{L}\end{array}$

91 AAG GAC CGT CAT GAC TTT GGA TTT CCA CAG GAG GAG TTT GGC AAC $\begin{array}{lllllllllllllll}\mathbf{K} & \mathbf{D} & \mathbf{R} & \mathbf{H} & \mathbf{D} & \mathbf{F} & \mathbf{G} & \mathbf{F} & \mathbf{P} & \mathbf{Q} & \mathbf{E} & \mathbf{E} & \mathbf{F} & \mathbf{G} & \mathbf{N}\end{array}$

136 CAG TTC CAA AAG GCT GAA ACC ATC CCT GTC CTC CAT GAG ATG ATC $\begin{array}{lllllllllllllll}\mathbf{Q} & \mathbf{F} & \mathbf{Q} & \mathbf{K} & \mathbf{A} & \mathbf{E} & \mathbf{T} & \mathbf{I} & \mathbf{P} & \mathbf{V} & \mathbf{L} & \mathbf{H} & \mathbf{E} & \mathbf{M} & \mathbf{I}\end{array}$

181 CAG CAG ATC TTC AAT CTC TTC AGC ACA AAG GAC TCA TCT GCT GCT $\begin{array}{lllllllllllllll}\mathbf{Q} & \mathbf{Q} & \mathbf{I} & \mathbf{F} & \mathbf{N} & \mathbf{L} & \mathbf{F} & \mathbf{S} & \mathbf{T} & \mathbf{K} & \mathbf{D} & \mathbf{S} & \mathbf{S} & \mathbf{A} & \mathbf{A}\end{array}$

226 TGG GAT GAG ACC CTC CTT GAC AAA TTC TAC ACT GAA CTC TAC CAG $\begin{array}{lllllllllllllll}\mathbf{W} & \mathbf{D} & \mathbf{E} & \mathbf{T} & \mathbf{L} & \mathbf{L} & \mathbf{D} & \mathbf{K} & \mathbf{F} & \mathbf{Y} & \mathbf{T} & \mathbf{E} & \mathbf{L} & \mathbf{Y} & \mathbf{Q}\end{array}$

271 CAG CTG AAT GAC CTG GAA GCC TGT GTG ATT CAG GGG GTG GGG GTG $\begin{array}{lllllllllllllll}\mathbf{Q} & \mathbf{L} & \mathbf{N} & \mathbf{D} & \mathbf{L} & \mathbf{E} & \mathbf{A} & \mathbf{C} & \mathbf{V} & \mathbf{I} & \mathbf{Q} & \mathbf{G} & \mathbf{V} & \mathbf{G} & \mathbf{V}\end{array}$

316 ACA GAG ACT CCA CTG ATG AAG GAG GAC TCC ATT CTG GCT GTG CGT

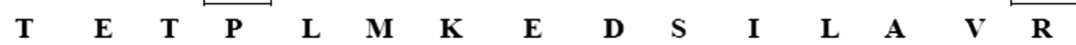

361 AAA TAC TTC CAA CGT ATC ACT CTC TAT CTG AAA GAG AAG AAA TAC $\begin{array}{lllllllllllllll}\mathbf{K} & \mathbf{Y} & \mathbf{F} & \mathbf{Q} & \mathbf{R} & \mathbf{I} & \mathbf{T} & \mathbf{L} & \mathbf{Y} & \mathbf{L} & \mathbf{K} & \mathbf{E} & \mathbf{K} & \mathbf{K} & \mathbf{Y}\end{array}$

406 AGC CCT TGT GCC TGG GAG GTT GTC CGT GCA GAA ATC ATG CGT TCT

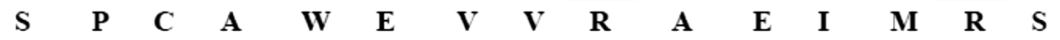

451 TTT TCT TTG TCA ACA AAC TTG CAA GAA AGT TTA CGT AGT AAG GAA

$$
\begin{array}{lllllllllllllll}
\mathbf{F} & \mathbf{S} & \mathbf{L} & \mathbf{S} & \mathbf{T} & \mathbf{N} & \mathbf{L} & \mathbf{Q} & \mathbf{E} & \mathbf{S} & \mathbf{L} & \mathbf{R} & \mathbf{S} & \mathbf{K} & \mathbf{E}
\end{array}
$$

496 GAC AAC GAG AAA TTA CGT AAA CCA AAA CAT AAA AAA TTA AAG CAA

$\begin{array}{lllllllllllllll}\mathbf{D} & \mathbf{N} & \mathbf{E} & \mathbf{K} & \mathbf{L} & \mathbf{R} & \mathbf{K} & \mathbf{P} & \mathbf{K} & \mathbf{H} & \mathbf{K} & \mathbf{K} & \mathbf{L} & \mathbf{K} & \mathbf{Q}\end{array}$

541 CCA GCG GAT GGT TAAT

$\begin{array}{lllll}\mathbf{P} & \mathbf{A} & \mathbf{D} & \mathbf{G} & -\end{array}$

Fig. $\mathbf{2}$ The IFN-CSP gene with codon optimization. The codons in frame are optimized

USA). The molecular weight of purified IFN-CSP was characterized by mass spectrometry on a matrix-assisted laser desorption/ionization (MALDI) mass spectrometer (Applied Biosystems, USA).

Biological activity of IFN-CSP was determined in a standard cytopathic effect inhibition assay using vesicular stomatitis virus (VSV)/human amniotic cells (WISH) measure system according to China Biologicals Requirements.
In vivo tissue distribution experiment

All animal experimental protocols were approved by the Guangdong Pharmaceutical University Animal Care and Use Committee. Normal Balb/c mice (18-22 g) were purchased from the Center for Experimental Animals of Guangdong Province (Guangzhou, China). Balb/c mice $(\mathrm{n}=90)$ were injected via the tail vein with $9.01 \mu \mathrm{g} / \mathrm{kg}$ $(0.46 \mathrm{nmol} / \mathrm{kg})$ of control IFN $\alpha 2 \mathrm{~b}$ or $10.09 \mu \mathrm{g} / \mathrm{kg}(0.46$

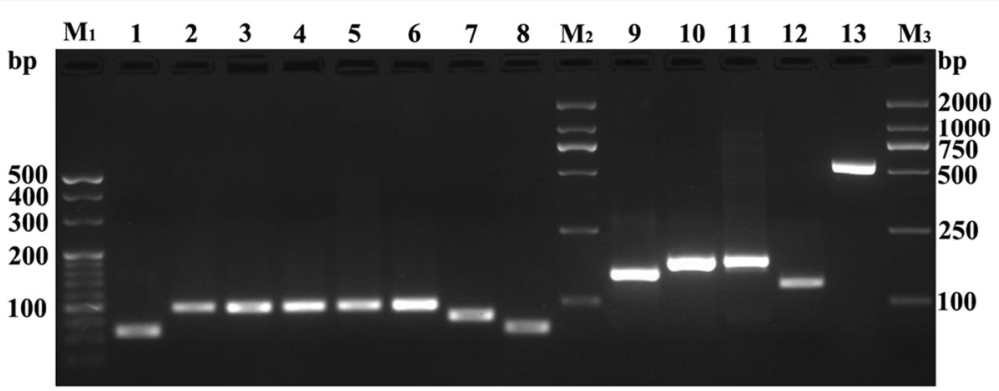

Fig. 3 Construction of fusion gene IFN-CSP. Lane M: DNA molecular weight marker, Lane 1-8: The first PCR products ranging 68-97 bp in size, Lane 9-12: The second PCR products ranging 132-175 bp in size, Lane 13: Target amplification the full-length fusion gene IFN-CSP 

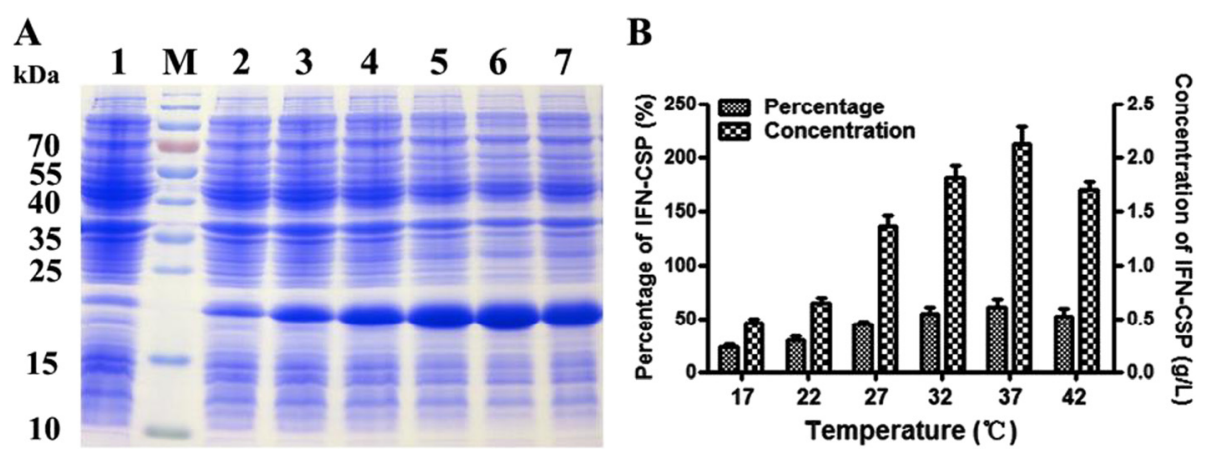

Fig. 4 Effect of temperature on the expression level of IFN-CSP in E. coli BL21/pET21b-IFN-CSP. a: SDS-PAGE analyses of IFN-CSP expression under different temperatures. Lane M: Protein molecular weight marker; Lane 1: E. coli BL21/pET21 b-IFN-CSP before induction; Lanes 2-7: E. coli BL21/ pET21 b-IFN-CSP was cultured at $17,22,27,32,37$ and $42^{\circ} \mathrm{C}$, respectively. $\mathbf{b}$ : Percentage and concentration of IFN-CSP were calculated by the target bands in SDS-PAGE (A)

$\mathrm{nmol} / \mathrm{kg}$ ) of recombinant IFN-CSP. At 30, 60, 120, 240 and $480 \mathrm{~min}$ post injection, the mice were anesthetized, euthanized and then the liver, heart, spleen, lung and kidney were excised, rinsed with saline, dried and weighed. The concentration of IFN $\alpha 2 b$ was determined by ELISA (Shanghai Senxiong Biotechnology Limited Corporation.) according to the manufacturer.

\section{In vivo anti-HBV experiment}

Balb/c-HBV transgenic mice (18-22 g) were purchased from Infectious Disease Center of No. 458 Hospital (Guangzhou, China) and kept under the pathogen free condition in the facility of the Guangdong Pharmaceutical University. The Balb/c-HBV transgenic mice were randomly assigned to either treatment or control groups $(n=6)$. Six non-transgenic Balb/c mice were used as normal control mice. Each mouse was intramuscular injected with IFN-CSP (at dose of $10^{1} \mathrm{U} / \mathrm{g}$ body weight, $10^{2} \mathrm{U} / \mathrm{g}$ body weight, $10^{3}$ $\mathrm{U} / \mathrm{g}$ body weight, respectively) once a day for 28 days. Sterile physiological saline was used as control. IFN $\alpha 2 \mathrm{~b}\left(10^{3} \mathrm{U} / \mathrm{g}\right.$ body weight) was used as drug control. Mice were sacrificed at day 28. Serum samples and livers were collected.

\section{Measurement of serum HBsAg and HBV-DNA}

Serum HBsAg was measured by enzyme-linked immunosorbent assay (ELISA) using a HBV diagnostic kit (Shanghai Kehua Biotech Co. Ltd., China) following the manufacturer's recommendations. Serum HBV-DNA was measured using a commercially available real-time fluorescent quantification PCR (FQ-PCR) diagnostic kit (Da-An Gene Co., Guangzhou, China) according to the manufacturer's instruction.

\section{Immunofluorescence and western blot analysis for HBV core protein}

Tissues were fixed in $4 \%$ paraformaldehyde for $1 \mathrm{~h}$, then in $30 \%$ sucrose solution overnight for cryoprotection. $10-12 \mu \mathrm{m}$ thick sections were cut using a Leica cryostat. Immunohistological detection of $\mathrm{HBcAg}$ was performed on frozen sections using primary rabbit polyclonal antiHBcAg antibody (1:200; Abcam, England) and Alexa Fluor 488-conjugated goat anti-rabbit IgG (1:200; Jackson ImmunoResearch, USA). Finally, the sections were stained with 4',6-diamidino-2-phenylindole (DAPI) for nuclear indication. Immunofluorescence analyses were performed with a fluorescence microscope (Leica, German).

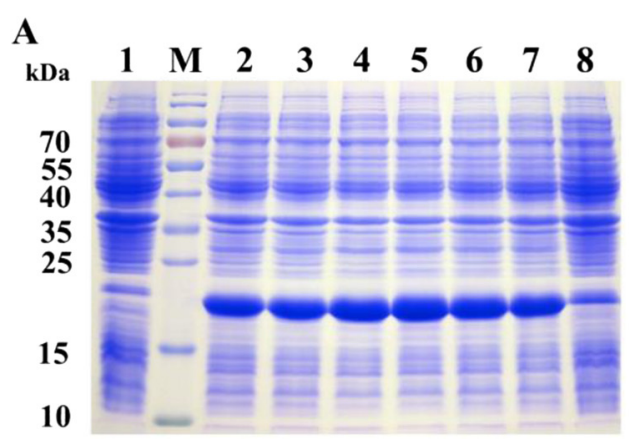

$\mathbf{B}$



Fig. 5 Effect of cell density on the expression level of IFN-CSP in E. coli BL21/pET21b-IFN-CSP. a: SDS-PAGE analyses of IFN-CSP expression at different growth stages. Lane M: Protein molecular weight marker; Lane 1: E. coli BL21/pET21b-IFN-CSP before induction; Lanes 2-8: E. coli BL21/pET21b-IFN-CSP was induced at $\mathrm{OD}_{600}$ value of $0.1,0.2,0.4,0.6,0.8,1.0$ and 2.0 , respectively. $\mathbf{b}$ : Percentage and concentration of IFN-CSP were calculated by the target bands in SDS-PAGE (A) 

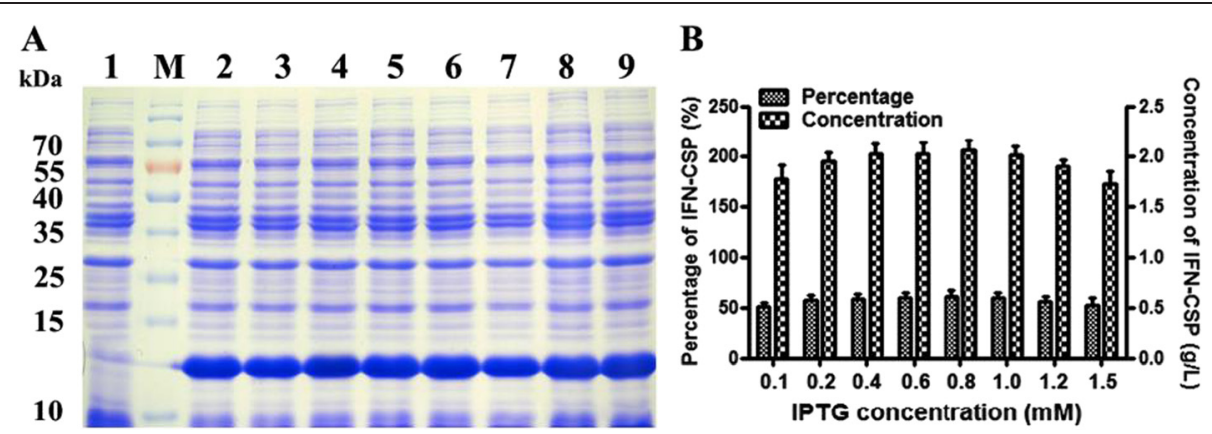

Fig. 6 Effect of different IPTG concentration on the expression level of IFN-CSP in E. coli BL21/pET21b-IFN-CSP. a: SDS-PAGE analyses of IFN-CSP expression using different IPTG concentration. Lane M: Protein molecular weight marker; Lane 1: E. coli BL21/pET21b-IFN-CSP before induction; Lanes 2-9: IPTG concentrations are $0.1,0.2,0.4,0.6,0.8,1.0,1.2$ and $1.5 \mathrm{mmol} / \mathrm{L}$, respectively. $\mathbf{b}$ : Percentage and concentration of IFN-CSP were calculated by the target bands in SDS-PAGE (A)

HBV core protein was also analyzed by a standard western blot procedure using primary polyclonal antiHBcAg antibody (1:200; Abcam, England), anti-actin monoclonal antibody (1:1000; Santa Cruz Biotechnology, USA) and peroxidase-conjugated secondary antibodies. The image was digitized using a scanner and signal was quantified using of Quantity One software (Bio-Rad).

\section{Statistical analysis}

Each measurement was performed at least in triplicate. All data were expressed as the mean \pm standard error of the mean (SEM). All statistical analyses were performed by SPSS (version 13.0 for Windows) statistical software. Differences between mean values were analyzed using One-Way Analysis of Variance (ANOVA). Statistical significance was defined by a $P$ value of less than 0.05 .

\section{Results}

Construction of the liver-targeting fusion interferon gene using $E$. coli preferred codon

To create a modified IFN-CSP gene with enhanced translation efficiency compared to the native gene, we designed a codon-optimized version of the IFN-CSP gene (GenBank Accession No. KP027473), which the rare codons of $E$. coli were changed to the preferential codons (Fig. 2). The new modified IFN-CSP gene has the identical amino acid sequence as the native gene. To increase the translation termination efficiency, the preferred stop codon TAAT was used. Finally, restriction enzyme sites for Nde I and Xho I were introduced at the 5' - and 3'-ends of the IFN-CSP gene to provide convenient restriction sites for cloning into the bacterial expression vector. Using SOE-PCR method, the synthetic IFN-CSP gene with the optimized codons was obtained (Fig. 3) and identified by DNA sequencing.

\section{Plasmids construction and protein expression}

The above synthetic IFN-CSP gene fragment was cloned into the bacterial expression vector pET-21b to construct the expression plasmid IFN-CSP/pET-21b (Fig. 1a). The correct synthetic sequence in the recombinant plasmid IFN-CSP/ pET-21b was identified by restriction enzymes with Nde I/Xho I, showing the expected about 600 bp DNA fragment (data not shown). The plasmid was then transformed into the bacterial expression host E.coli BL21 (DE3) to obtain recombinant strain. The target protein, which about $21.5 \mathrm{kD}$, was successfully expressed (Fig. 1b) in the form of inclusion bodies (IB).

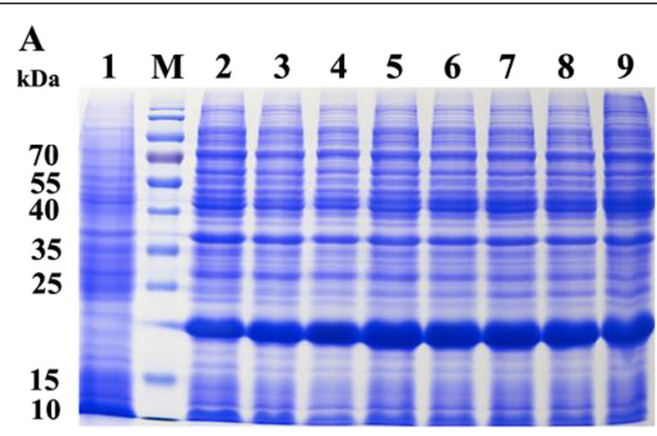

$\mathbf{B}$

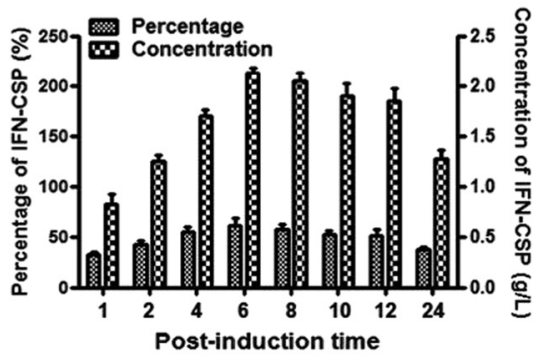

Fig. 7 Effect of post-induction time on the expression level of IFN-CSP in E. coli BL21/pET21b-IFN-CSP. a: SDS-PAGE analyses of IFN-CSP expression at different post-induction time. Lane M: Protein molecular weight marker; Lane 1: E. coli BL21/pET21 b-IFN-CSP before induction; Lanes 2-9: E. coli BL21/pET21 b-IFN-CSP after induced 1, 2, 4, 6, 8, 10, 12 and 24 h, respectively. $\mathbf{b}$ : Percentage and concentration of IFN-CSP were calculated by the target bands in SDS-PAGE (A) 


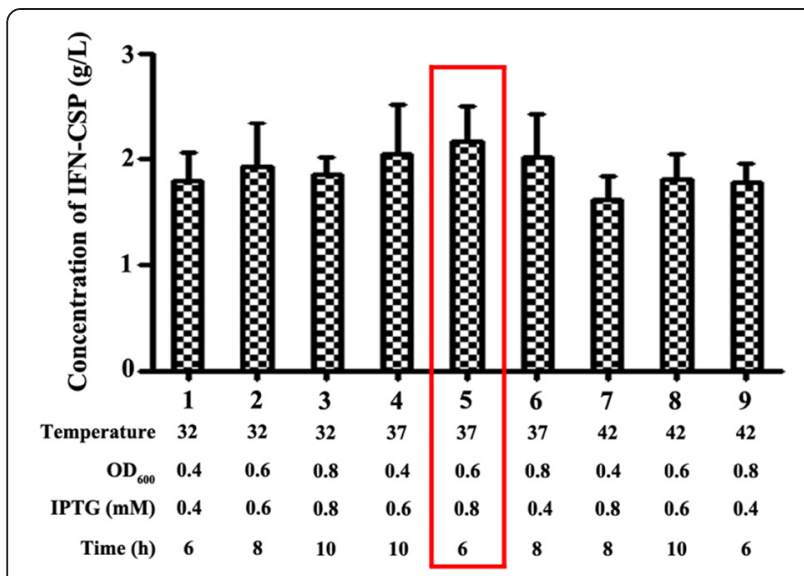

Fig. 8 Optimization of IFN-CSP expression by orthogonal test

The effects of different expression conditions

Several one-factor experiments and an orthogonal test $(\mathrm{L}(9)(3)(4))$ were employed to optimize the induction conditions.

\section{Cultivation temperature}

It is well-known that temperature has a great impact on the recombinant protein expression. Effects of temperature on IFN-CSP expression were investigated at $17,22,27,32,37$ and $42{ }^{\circ} \mathrm{C}$, respectively. The results (Fig. 4) revealed that IFN-CSP achieved a high percentage in total proteins and a high concentration in the temperature range of $32-37^{\circ} \mathrm{C}$.

\section{Induction timing}

IPTG induction initiates the translation of heterologous protein. The effect of induction timing was evaluated by adding IPTG at different stages of growth phase. The results (Fig. 5) revealed that the percentage of target protein to total proteins varied from 20.12 to $61.62 \%$ at different induction timing. The maximum percentage was observed when induced at the midexponential phase, corresponding to the $\mathrm{OD}_{600}$ value of $0.4-0.8$.

\section{IPTG concentration}

Effects of final IPTG concentration on IFN-CSP expression were investigated at $0.1,0.2,0.4,0.6,0.8,1.0,1.2$ and $1.5 \mathrm{mM}$, respectively. The results (Fig. 6) revealed that the percentage of target protein in the total proteins and the concentration of IFN-CSP achieved the highest level in the IPTG range of $0.4-0.8 \mathrm{mM}$.

\section{Post-induction time}

The results of optimal post-induction time (Fig. 7) revealed that the percentage of IFN-CSP to total proteins varied from $32.55 \%$ to $60.89 \%$ at different post-induction time. The maximum percentage was observed the product reached the highest concentration after a 6-10 h induction.

\section{Orthogonal test}

Based on the results of one-factor experiments, four parameters were further optimized by an orthogonal test $(\mathrm{L}(9)(3)(4))$. The result of orthogonal test was showed that

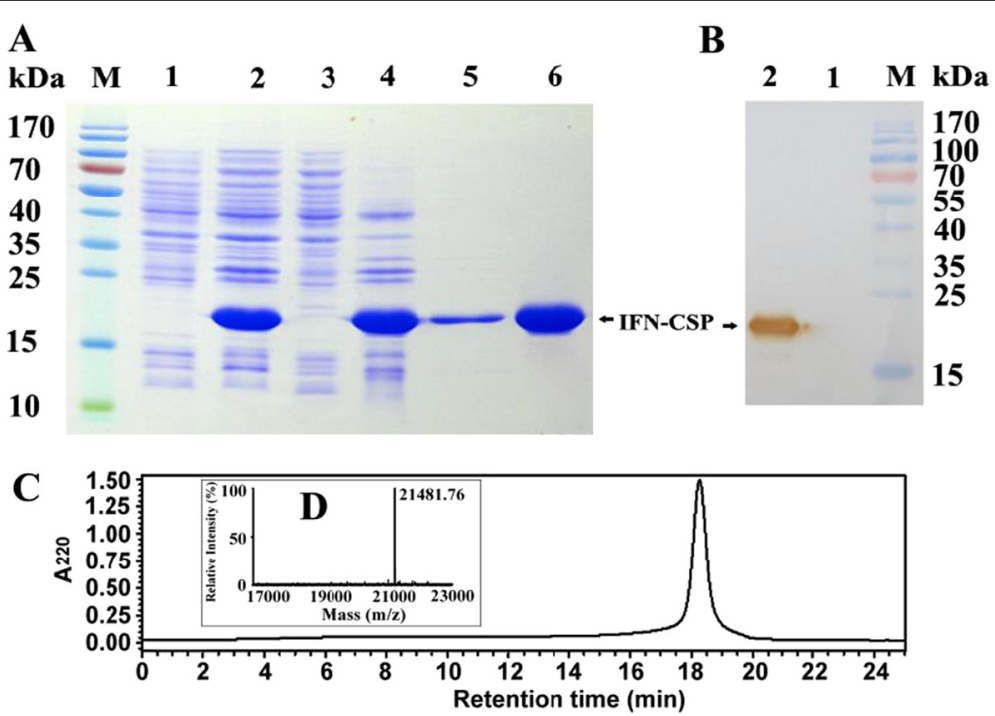

Fig. 9 Characterization of recombinant protein by SDS-PAGE, western blot, RP-HPLC and MALDI-MS. a: Purification of IFN-CSP. Lane M: Protein molecular weight marker, Lane 1-2: Total proteins of E. coli BL21/pET-21b-IFN-CSP before and after induction, Lane 3-4: Supernatant and precipitation after ultrasonication and centrifugation. Lane 5: Purified IFN-CSP using trion and urea wash. Lane 6: Purified IFN-CSP using HiTrap affinity chromatography. b: IFN-CSP was analyzed by western blot. Lane M: Protein molecular weight marker. Lane 1-2: Total proteins of E. coli BL21/pET-21b-IFN-CSP before and after induction. c: Analysis of purified IFN-CSP by RP-HPLC with a C18 column. d: Mass spectrum of purified IFN-CSP recorded on an Applied Biosytems Voyager MALDI-TOF mass spectrometry 
Table 2 Isolation of IFN-CSP from E. coli inclusion bodies with a recovery protocol

\begin{tabular}{lllll}
\hline Process step & $\begin{array}{l}\text { Total protein } \\
(\mathrm{mg} / \mathrm{L})\end{array}$ & $\begin{array}{l}\text { Purity } \\
(\%)\end{array}$ & $\begin{array}{l}\text { IFN-CSP } \\
(\mathrm{mg} / \mathrm{L})\end{array}$ & $\begin{array}{l}\text { Recovery yield } \\
(\%)\end{array}$ \\
\hline Cell lysis & 3512 & 61.62 & 2164.09 & 100.00 \\
Centrifugation & 2536 & 79.09 & 2005.72 & 92.68 \\
Triton wash + Urea wash & 1894 & 85.97 & 1628.27 & 81.18 \\
Solubilization & 1690 & 86.39 & 1459.99 & 89.60 \\
Refolding & 1359 & 87.16 & 1184.50 & 81.13 \\
HiTrap affinity & 735 & 99.85 & 733.89 & 61.96 \\
Dialysis & 691 & 99.85 & 690.00 & 93.73 \\
\hline
\end{tabular}

Total protein concentration was determined by Bradford method. Purity of IFN-CSP was calculated from densitometry analysis of SDS-PAGE gels. The concentration of IFN-CSP was calculated according the percentage fraction and total protein concentration the optimal condition of maximum expression quantity was inducing the culture at $\mathrm{OD}_{600}=0.6$ with $0.8 \mathrm{mM}$ IPTG for $6 \mathrm{~h}$ at $37^{\circ} \mathrm{C}$. The target protein was up to $2.17 \mathrm{~g}$ per L culture (Fig. 8). Main influence factor was induction temperature (data not shown).

\section{Purification, antigenicity and biological activity analysis}

The total improved isolation scheme was showed in Fig. 9. The results of SDS-PAGE and RP-HPLC revealed that the target protein was purified to over $95 \%$ homogeneity with no degradation (Fig. 9a and c). The molecular weight 21,481.76 Da determined by MALDI-MS (Fig. 9d) was consistent with its calculated value of $21,481.7$ Da. Step yields were calculated and showed in Table 2. The improved isolation scheme were highly efficient in producing pure IFN-CSP, approximately $690 \mathrm{mg}$ of the pure recombinant IFN-CSP was obtained from $1 \mathrm{~L}$ of $E$. coli culture.

The results of western blot analysis revealed that purified IFN-CSP was strongly and specifically reacted with the IFN $\alpha$ antibody (Fig. 9b). The results of chromogenic

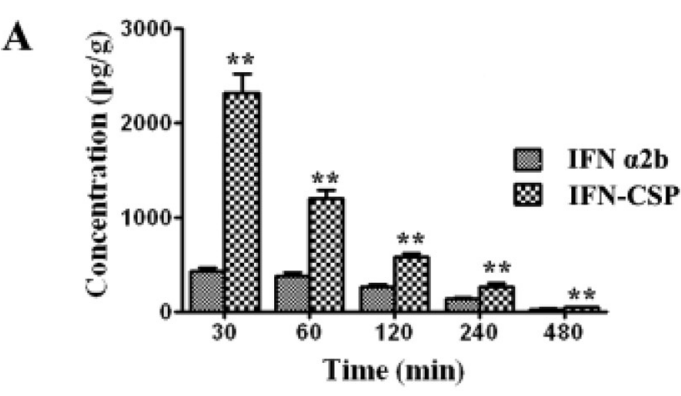

B

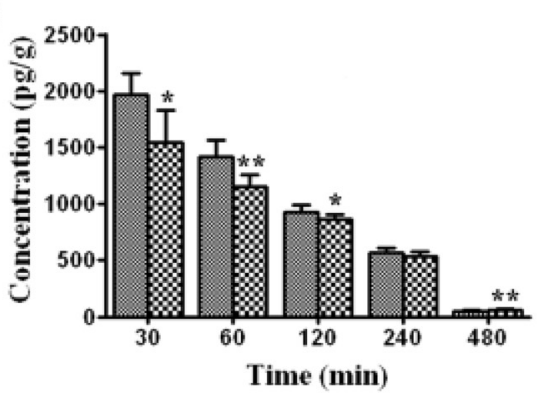

D

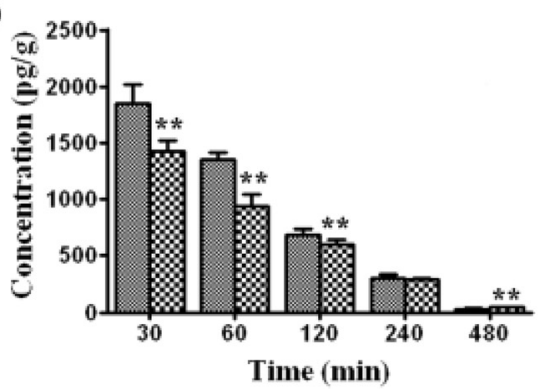

C

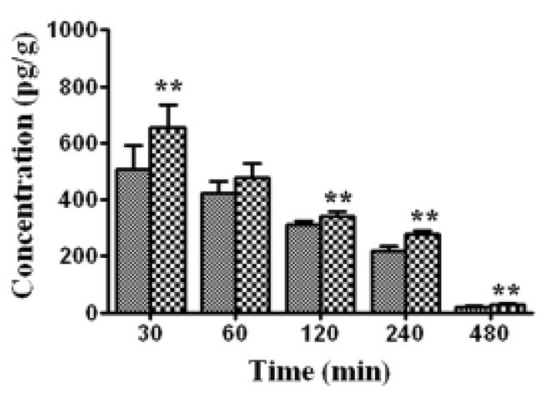

$\mathbf{E}$

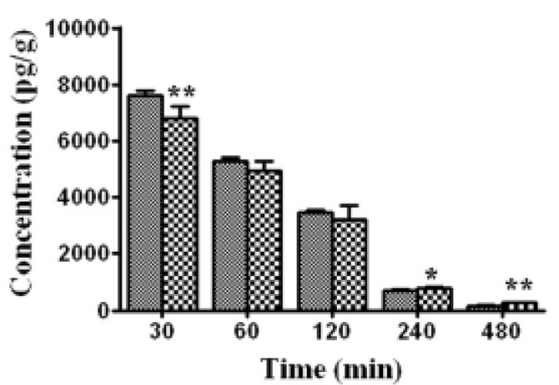

Fig. 10 Distribution of IFN a2b and IFN-CSP in liver (a), heart (b), spleen (c), lung (d), kidney (e) after a single i.V. administration of free IFN a2b or IFN-CSP in mice at 30, 60, 120, 240 and 480 min post-injection. Each point represents the mean \pm SEM $(n=6)$. The ** and * indicate statistically significant differences of $p<0.01$ and $p<0.05$, respectively between IFN $\mathrm{a} 2 \mathrm{~b}$ and IFN-CSP treatments 

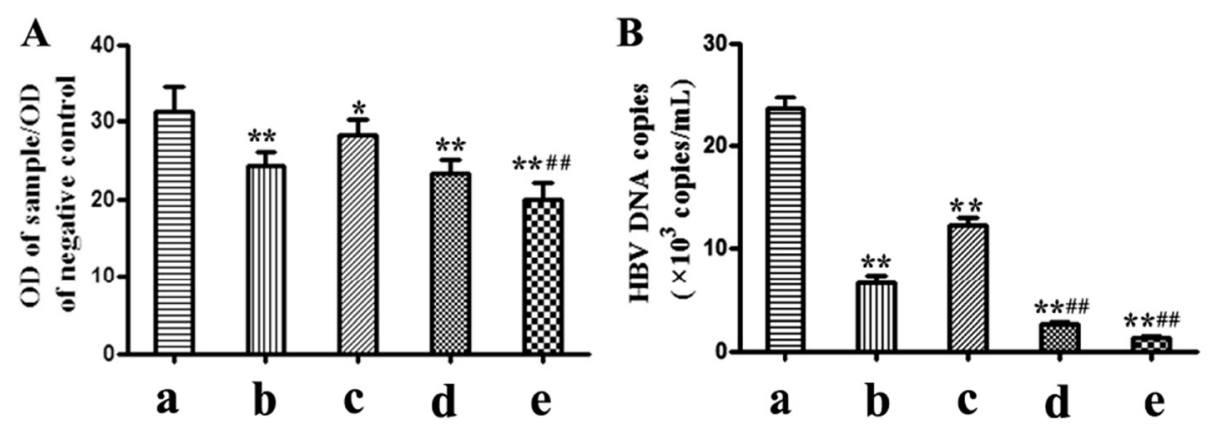

Fig. 11 Effect of IFN a2b, IFN-CSP on serum HBsAg (a) and HBV-DNA (b) level in HBV-transgenic mice. (a) physiological saline control; (b) IFN a2b, $10^{3} \mathrm{U} / \mathrm{g}$ body weight; (c) IFN-CSP, $10^{1} \mathrm{U} / \mathrm{g}$ body weight; (d) IFN-CSP, $10^{2} \mathrm{U} / \mathrm{g}$ body weight; (e) IFN-CSP, $10^{3} \mathrm{U} / \mathrm{g}$ body weight. The treatment continued for 28 days, serum HBsAg levels were measured by ELISA assay. The results are represented as OD value of sample/OD value of negative control. Serum HBV-DNA levels were measured by fluorescent quantification polymerase chain reaction (PCR). The copies of the HBV-DNA were calculated based on their $\mathrm{Ct}$ value and the standard curves. The data are the mean \pm SEM from six mice. ${ }^{*} P<0.05$ vs physiological saline controls, ${ }^{*} P<0.01$ vs physiological saline controls, ${ }^{\#} P<0.01$ vs IFN a2b controls

limulus amoebocyte lysate assay showed that the LPS content in the purified sample was less than $0.5 \mathrm{Eu} / \mathrm{mg}$ proteins. Biological activity analysis using the WISH/ VSV system showed that the above purified sample had ability to inhibit the cytopathic effect caused by VSV on WISH cells $\left(3.78 \times 10^{8} \mathrm{U} / \mathrm{mg}\right)$.

\section{In vivo tissue distribution}

To determine whether IFN-CSP was able to accumulate in liver for potential treatment of hepatitis $B$, the tissue distribution of IFN-CSP were evaluated and compared to native IFN $\alpha 2 b$. The ELISA method for IFN $\alpha 2 b$ detection is feasible and accurate (data not shown). The IFN-
A
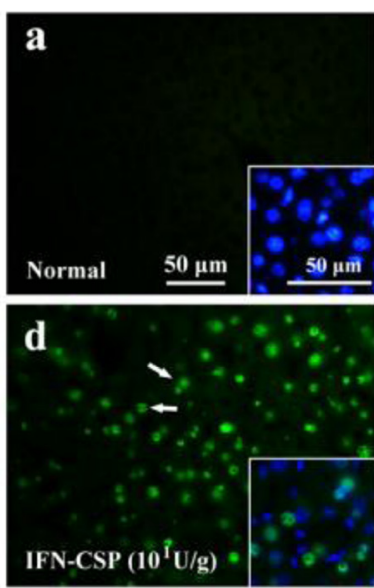

B

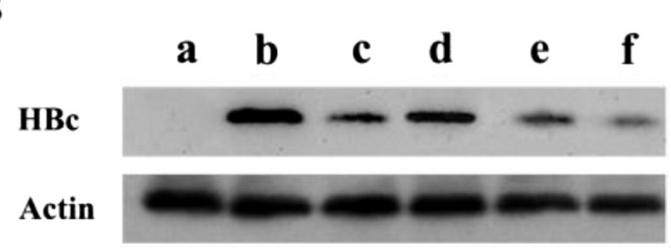


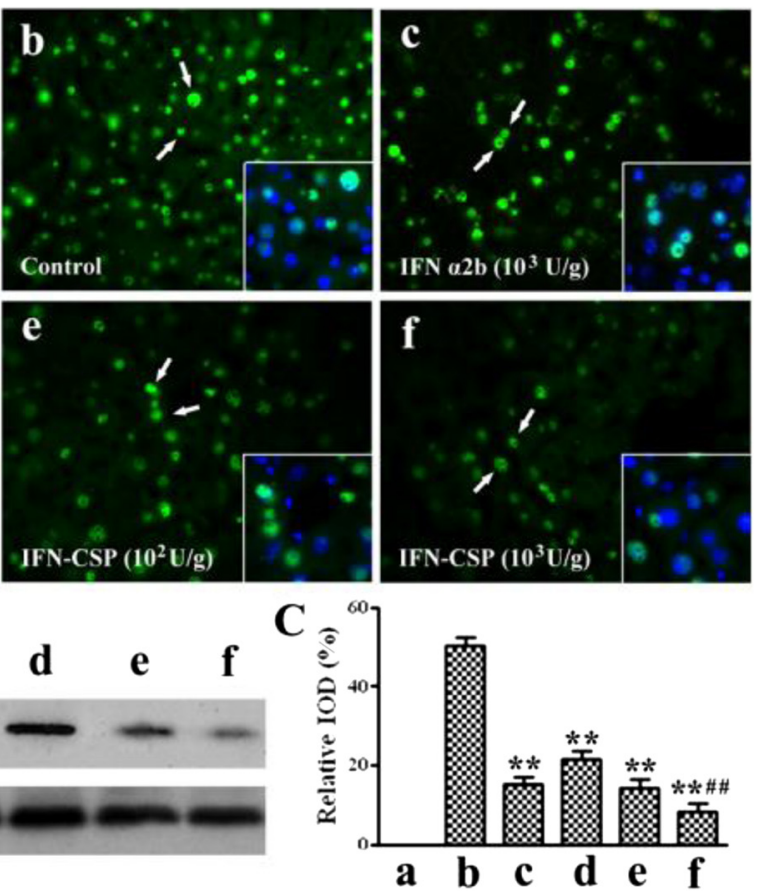

Fig. 12 Effect of IFN a2b, IFN-CSP on HBV core protein in the liver of HBV-transgenic mice. $\boldsymbol{a}$ normal non-transgenic mice control; (b) physiological saline control; (c) IFN a2b, $10^{3} \mathrm{U} / \mathrm{g}$ body weight; (d) IFN-CSP, $10^{1} \mathrm{U} / \mathrm{g}$ body weight; (e) IFN-CSP, $10^{2} \mathrm{U} / \mathrm{g}$ body weight; (f) IFN-CSP, $10^{3} \mathrm{U} / \mathrm{g}$ body weight. The treatment continued for 28 days, liver of mice were collected for analyses. a: Representative photographs of liver sections immunofluorescent stained with anti-HBCAg antibody are presented. Arrows indicate distinct green fluorescent labeling of HBcAg in liver, blue nuclear stained with DAPI. Calibration bar $=50 \mu \mathrm{m}$ for photomicrographs. $\mathbf{b}$ : The core protein was also quantified by western blot analysis. c: Optical densities of the core protein were analyzed using Quantity One software. The data are the mean \pm SEM from six mice. ${ }^{*} P<0.05$ vs physiological saline controls, ${ }^{* *} P<0.01$ vs physiological saline controls, ${ }^{\#}{ }^{\#} P<0.01$ vs IFN a2b controls 
CSP increased the liver IFN $\alpha 2 b$ levels more significantly, up to 5.48- to 3.22-fold higher than those of native IFN $\alpha 2 \mathrm{~b}(p<0.01)$ at 30 , and $60 \mathrm{~min}$ interval postadministration (Fig. 10).

\section{IFN-CSP reduces serum $\mathrm{HBsAg}$ and $\mathrm{HBV}-\mathrm{DNA}$ in HBV-transgenic mice}

To evaluate the in vivo anti-HBV activity of the recombinant IFN-CSP, Balb/c-HBV transgenic mice were treated with different concentrations of IFN-CSP for 28 days. As shown in Fig. 11, IFN-CSP reduces serum HBsAg and HBV-DNA in HBV-transgenic mice in a dose-dependent manner. Compared with the native IFN $\alpha 2 b$ group, the administration of IFN-CSP at comparable concentrations significantly reduced serum HBsAg and HBV-DNA.

\section{IFN-CSP reduces $\mathrm{HBV}$ core protein in the liver of HBV-transgenic mice}

Livers from HBV-transgenic mice were detected with anti-HBcAg antibody by immunofluorescent staining and western blot analysis. The results (Fig. 12) showed that the signal of HBV core protein is obviously decreased in the liver of HBV-transgenic mice treated with IFN-CSP in a dose-dependent manner. IFN $\alpha 2 \mathrm{~b}$ control also suppressed the expression of HBV core protein, but the degree of suppression less than the same dose of IFN-CSP.

\section{Discussion}

The expression of heterologous proteins in E. coli is an attractive strategy to obtain the active form of protein in a large scale for therapeutic application. In our previous work, the strategy proved to be efficient to achieve highlevel expression $[15,17]$. While, DNA sequence analysis of genes encoding either high abundance or low abundance proteins in $E$. coli has revealed a pattern of favored codon usage [18]. Lowly expressed genes show the greatest degree of conformity to the rare codons, which correspond to the rare tRNAs in the E. coli cell. So, it is necessary to analyze the DNA sequence of IFN-CSP before recombination expression using $E$. coli expression system.

The IFN-CSP contains 184 amino acids, and 15 amino acids are encoded by the rare codons of E. coli. Particularly, R12, R13, R22, R23, R33, R120, R125, R144, R149, and R162 were encoded by the least used codons, AGG and AGA, which were discovered to dramatically reduce the maximum level of protein synthesis. Some examples show that codon optimization can significantly improve the expression level $[5,19]$. Srivastava et al. reported that a codon optimized IFN $\alpha 2 b$ gene expressed as IB only about $40 \%$ of the total protein [20]. Valente et al. using an artificial IFN $\alpha 2 b$ gene that was codon optimized for its rare tandem arginine codons and its gene product was produced $16.6 \mathrm{mg} / \mathrm{L} \mathrm{E}$. coli culture [21]. Another investigation used $E$. coli codon optimized artificial gene produced His-IFN $\alpha 2 b$ in the form of IB $-210 \mathrm{mg} / \mathrm{L} E$. coli culture [22]. Based on the above knowledge, we applied a codon optimization strategy to over-express IFNCSP fusion protein in E. coli. Moreover, stop codon bias and context also correlates with the efficiency of translation [23], so the preferred sequence of translation termination in E. coli, 'TAAT' was used.

The induction conditions will affect the expression level of target proteins as reported numerous times elsewhere $[16,24]$. For this reason, the induction conditions like cultivation temperature, induction timing, inducer concentrations, and induction time were also examined. In our previous study, we found that the fusion IFN-CSP without any optimization expressed as IB only accounting for $39.2 \%$ of the total insoluble protein [13]. In the present, the highest expression level was achieved when the recombinant $E$. coli at the mid-exponential phase was effectively induced at $37^{\circ} \mathrm{C}$ with $0.8 \mathrm{mM}$ IPTG concentration for $6 \mathrm{~h}$. Although most of the IFN-CSP protein in the current work was produced in the form of IB (Fig. 1b), this

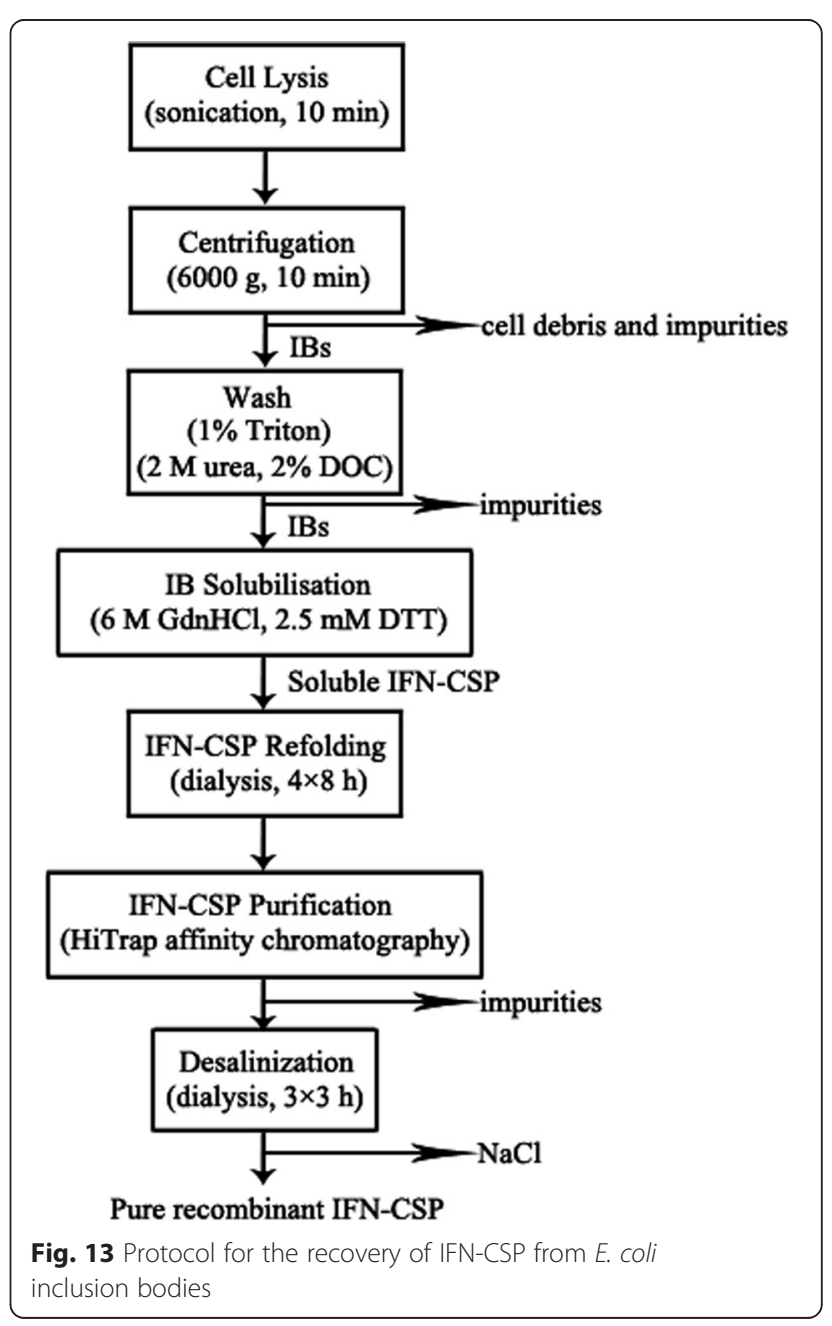


is the first report to show that the novel liver-targeting fusion interferon (IFN-CSP) was expressed in such high level, about $61.62 \%$ relative to total host proteins. Using the improved purification scheme (Fig. 13), the final yield of purified IFN-CSP was up to $690 \mathrm{mg}$ per L culture, which is 2.56-fold higher than the previous investigation [13]. The purity and the molecular weight of purified IFN-CSP were demonstrated by SDS-PAGE, RP-HPLC and MALDI-MS (Fig. 9). The result of western blot analysis characterized the antigenicity of IFN-CSP (Fig. 9b). Biological activity analysis according to China Biologicals Requirements showed that IFN-CSP had ability to inhibit the cytopathic effect caused by VSV on WISH cells $\left(3.78 \times 10^{8}\right.$ $\mathrm{U} / \mathrm{mg}$ ).

In line with previous in vitro experiments, we have further evaluated the in vivo tissue distribution and antiHBV activities of IFN-CSP. In vivo tissue distribution assay showed that IFN-CSP was able to accumulate in mouse liver compared to native IFN $\alpha 2 b$ (Fig. 10). Small animal models amenable to infection with human hepatotropic viruses are in great need for studying new anti$\mathrm{HBV}$ drugs. The HBV-Tg mouse lineage was initially produced on a BALB/c background. The transgene in these mice consists of 1.3 copies of the HBV ayw complete genome. The HBV-Tg mice express high level of HBsAg in their serum and have detectable HBV-DNA in their serum, which allows HBV-DNA replication, is an appropriate tool to evaluate anti-HBV drugs [25-28]. In the present study, we found that the recombinant IFN-CSP and native IFN $\alpha 2 b$ in vivo were able to reduce serum HBsAg (Fig. 11a), HBV-DNA (Fig. 11b) and liver HBV core protein (Fig. 12) in HBV-transgenic mice. However, IFN-CSP showed a significant improvement in anti-HBV activity when compared with native IFN $\alpha 2 b$ at similar doses. These results indicate that incorporation of plasmodium region I peptide can specifically target to liver, to achieve higher local concentrations and to improve their anti-HBV efficacy in vivo.

\section{Conclusion}

In this report, synthetic codon optimized IFN-CSP gene was overexpressed at high expression level and the purified protein was confirmed to IFN-CSP based on the results of antigenicity and biological activity analysis. The purified IFN-CSP was able to accumulate in mouse liver compared to native IFN $\alpha 2 b$ and reduce serum HBsAg, HBV-DNA and liver HBV core protein in HBV-transgenic mice. This current work reports for the first time that codon and expression conditions optimized approach is a strategy to produce IFN-CSP at large scale, and would also be very helpful to produce other recombinant protein for further medical research. The present study further supported the application of IFN-CSP in liver-targeting antiHBV medicines.

\section{Abbreviations}

IFN-CSP: Liver-targeting fusion interferon (fusing interferon with circumsporozoite protein region I-plus); HBV: Hepatitis B virus; E. coli: Escherichia coli; IPTG: Isopropyl B-D-1-thiogalactopyranoside; IFNs: Interferons; PCR: Polymerase chain reaction; SOE-PCR: Splicing by overlapping extension-PCR; LB: Luria-Bertani; 6-His: 6-histidine; SDS-PAGE: Sodium dodecyl sulphate polyacrylamide gel electrophoresis; GuHCl: Guanidine hydrochloride; LPS: Lipopolysaccharides; VSV: Vesicular stomatitis virus; WISH: Human amniotic cells; HBsAg: Hepatitis B s antigen; HBCAg: Hepatitis B core antigen; ELISA: Enzyme-linked immunosorbent assay; FQ-PCR: Fluorescent quantification PCR; DAPI: 4',6-diamidino-2phenylindole; SEM: Standard error of the mean; ANOVA: Analysis of Variance; IB: Inclusion bodies.

\section{Competing interests}

The authors declare that they have no competing interests.

\section{Authors' contributions}

$\mathrm{XL}$, JW and XJ performed gene construction, protein expression studies and animal experiment; $\mathrm{XL}$ and $J \mathrm{Z}$ designed the experiments, analyzed data, and wrote the paper. All authors read and approved the final manuscript.

\section{Acknowledgements}

This work was financially supported by National Major Scientific and Technological Special Project for "Significant New Drugs Development" of China (2013ZX09103003-003).

Received: 20 March 2015 Accepted: 29 May 2015

Published online: 12 June 2015

\section{References}

1. Kim CK, Choi JH, Lee SB, Lee SM, Oh JW. Expression and purification of recombinant human granulocyte colony-stimulating factor in fed-batch culture of Escherichia coli. Appl Biochem Biotechnol. 2014;172:2425-35.

2. Naz F, Asad M, Malhotra P, Islam A, Ahmad F, Hassan Ml. Cloning, expression, purification and refolding of microtubule affinity-regulating kinase 4 expressed in Escherichia coli. Appl Biochem Biotechnol. 2014;172:2838-48

3. Cao H, Lin R, Ghosh S, Anderson RA, Urban Jr JF. Production and characterization of ZFP36L1 antiserum against recombinant protein from Escherichia coli. Biotechnol Prog. 2008;24:326-33.

4. Qian B, Shen H, Xiong J, Chen L, Zhang L, Jia J, et al. Expression and purification of the synthetic preS1 gene of Hepatitis B Virus with preferred Escherichia coli codon preference. Protein Expr Purif. 2006:48:74-80.

5. Xu Z, Zhong Z, Huang L, Peng L, Wang F, Cen P. High-level production of bioactive human beta-defensin-4 in Escherichia coli by soluble fusion expression. Appl Microbiol Biotechnol. 2006;72:471-9.

6. Bandurska K, Krol I, Myga-Nowak M. Interferons: between structure and function. Postepy Hig Med Dosw (Online). 2014;68:428-40.

7. Cho H, Kelsall BL. The role of type I interferons in intestinal infection, homeostasis, and inflammation. Immunol Rev. 2014;260:145-67.

8. Belloni L, Allweiss L, Guerrieri F, Pediconi N, Volz T, Pollicino T, et al. IFN-alpha inhibits HBV transcription and replication in cell culture and in humanized mice by targeting the epigenetic regulation of the nuclear cccDNA minichromosome. J Clin Invest. 2012;122:529-37.

9. Retnoningrum DS, Ningrum RA, Kurniawan YN, Indrayati A, Rachmawati H. Construction of synthetic open reading frame encoding human interferon alpha $2 \mathrm{~b}$ for high expression in Escherichia coli and characterization of its gene product. J Biotechnol. 2010;145:193-8.

10. Suginoshita Y, Tabata Y, Moriyasu F, Ikada Y, Chiba T. Liver targeting of interferon-beta with a liver-affinity polysaccharide based on metal coordination in mice. J Pharmacol Exp Ther. 2001;298:805-11.

11. Longmuir KJ, Robertson RT, Haynes SM, Baratta UL, Waring AJ. Effective targeting of liposomes to liver and hepatocytes in vivo by incorporation of a Plasmodium amino acid sequence. Pharm Res. 2006;23:759-69.

12. Longmuir KJ, Haynes SM, Baratta JL, Kasabwalla N, Robertson RT. Liposomal delivery of doxorubicin to hepatocytes in vivo by targeting heparan sulfate. Int J Pharm. 2009:382:222-33.

13. Lu XM, Jin XB, Huang YT, Wang J, Shen J, Chu FJ, et al. Construction of a novel liver-targeting fusion interferon by incorporation of a Plasmodium region I-plus peptide. BioMed Res. 2014;2014:e261431. 
14. Lu XM, Wang J, Jin XB, Huang YT, Zeng WT, Zhu JY. IFN-CSP inhibiting hepatitis B virus in HepG2.2.15 cells involves JAK-STAT signal pathway. BioMed Res. 2015;2015:e959684.

15. Lu XM, Jin XB, Zhu JY, Mei HF, Ma Y, Chu FJ, et al. Expression of the antimicrobial peptide cecropin fused with human lysozyme in Escherichia coli. Appl Microbiol Biotechnol. 2010:87:2169-76.

16. Liu JF, Zhang ZJ, Li AT, Pan J, Xu JH. Significantly enhanced production of recombinant nitrilase by optimization of culture conditions and glycerol feeding. Appl Microbiol Biotechnol. 2011;89:665-72.

17. Shen J, Lu XM, Jin XB, Ding J, Li XB, Mei HF, et al. Expression of a novel dual-functional protein-the antimicrobial peptide LL-37 fused with human acidic fibroblast growth factor in Escherichia coli. Protein Expr Purif. 2012:81:119-25.

18. Sharp PM, Li WH. The codon Adaptation Index-a measure of directional synonymous codon usage bias, and its potential applications. Nucleic Acids Res. 1987;15:1281-95.

19. Peng L, Xu Z, Fang X, Wang F, Yang S, Cen P. Preferential codons enhancing the expression level of human beta-defensin-2 in recombinant Escherichia coli. Protein Pept Lett. 2004;11:339-44.

20. Srivastava P, Bhattacharaya P, Pandey G, Mukherjee KJ. Overexpression and purification of recombinant human interferon alpha2b in Escherichia coli. Protein Expr Purif. 2005;41:313-22.

21. Valente CA, Prazeres DM, Cabral JM, Monteiro GA. Translational features of human alpha 2b interferon production in Escherichia coli. Appl Environ Microbiol. 2004;70:5033-6.

22. Neves FO, Ho PL, Raw I, Pereira CA, Moreira C, Nascimento AL. Overexpression of a synthetic gene encoding human alpha interferon in Escherichia coli. Protein Expr Purif. 2004:35:353-9.

23. Sun J, Chen M, Xu J, Luo J. Relationships among stop codon usage bias, its context, isochores, and gene expression level in various eukaryotes. J Mol Evol. 2005;61:437-44

24. Yan X, Hu S, Guan YX, Yao SJ. Coexpression of chaperonin GroEL/GroES markedly enhanced soluble and functional expression of recombinant human interferon-gamma in Escherichia coli. Appl Microbiol Biotechnol. 2012:93:1065-74.

25. Gao LF, Sun WS, Ma CH, Liu SX, Wang XY, Zhang LN, et al. Establishment of mice model with human viral hepatitis B. World I Gastroenterol. 2004;10:841-6.

26. Li D, Xu DZ, Choi BC, Men K, Zhang JX, Lei XY, et al. Preliminary study on the efficacy and safety of lamivudine and interferon alpha therapy in decreasing serum HBV DNA level in HBV positive transgenic mice during pregnancy. J Med Virol. 2005;76:203-7.

27. Cobleigh MA, Wei X, Robek MD. A vesicular stomatitis virus-based therapeutic vaccine generates a functional CD8 T cell response to hepatitis $B$ virus in transgenic mice. J Virol. 2013;87:2969-73

28. Tang Y, Chen X, Zhang Y, Tang Z, Zhuo M, Li D, et al. Fusion protein of tapasin and hepatitis B core antigen 1827 enhances $T$ helper cell type 1/2 cytokine ratio and antiviral immunity by inhibiting suppressors of cytokine signaling family members $1 / 3$ in hepatitis B virus transgenic mice. Mol Med Rep. 2014:9:1171-8.

\section{Submit your next manuscript to BioMed Central and take full advantage of:}

- Convenient online submission

- Thorough peer review

- No space constraints or color figure charges

- Immediate publication on acceptance

- Inclusion in PubMed, CAS, Scopus and Google Scholar

- Research which is freely available for redistribution 